\title{
Mathematical Models of Knowledge and Skill Accumulating in the Process of Learning
}

\author{
Zhong-Lin Chai \\ College of Sciences, China Jiliang University \\ Hangzhou, P.R. China \\ chaizhonglin@163.com
}

\begin{abstract}
Education is usually for recipient to learn. Learning mainly is that one learns knowledge and/or skills. Thus, it is very meaningful to study how a learner accumulates knowledge and skill in learning process. This paper establishes several mathematical models of knowledge and skill accumulated in learning process, and then discusses the correctness of the models. After that it uses the models to discuss the two common learning modes, namely school-learning and self-learning. It points out the advantages and disadvantages of the two modes, and shows that school-learning is the most effective learning pattern for ordinary people. Besides, it also shows the instructive meaning of the models for educators and learners through several situations.
\end{abstract}

Keywords-Learning; School-learning; Self-learning; Knowledge and skill; Model

\section{INTRODUCTION}

With the development of science and technology learning is becoming more and more important, and the time spent in learning for one in his life is continuously increasing. For example in China, it takes about sixteen years for one to learn from elementary school to college graduation. Even if he works after leaving school, for the need of livelihood and/or mastering new knowledge and skills emerging constantly, he still needs to take part in various career-trainings and self-learning. So learning has become a lifelong need for people $[1,2]$. Where there is learning, there are learning styles, and there is a need to study the efficiency of different learning patterns so as to select the best or better one. There are two common or main learning patterns, school-learning and self-learning. With the lifelong need for people to learn in modern society and the development of communication tools such as Internet, and to make up the defects of school-learning and self-learning, many new learning styles were presented, such as blended learning [3], autonomic learning [4] and e-learning [5]. The investigations of these styles undoubtedly promote the development of learning and education theory. But these studies usually are qualitative, rarely from quantitative angle through complete mathematical models. In fact, the aim of learning usually is to get the knowledge and skills contained in the learning goal, and the learning process is just a procedure of one accumulating knowledge and skill constantly. Based on it, this paper aims to build mathematical models of knowledge and skill accumulating in learning process so as to investigate learning phenomenon from quantitative perspective. Besides, the models will give some enlightenment for educators and learners.

\section{CONCEPTS EXPLANATION AND SYMBOLS EXPLICATION}

For the sake of convenience of building models, here gives the explanations of some concepts and symbols to be used in the following.

Learning goal: simply called goal. The goals set by different people in learning have different formats and contents, but all are related to knowledge and skill. Here a goal is defined as the following: one intends to master some knowledge and skills with somewhat integrality and systematicness.

Learning resource: all resources available for a learner for the learning of the goal, which include the related books, equipments as well as whether the learner has chances to communicate with those experienced people, etc. Obviously the requirements for resource are different for different goals.

Learning strategy: all methods a learner uses for the learning of the goal. They include not only various specific learning methods, but also the orders arrangement of learning different parts of the knowledge and skills of the goal as well as the time arrangement of learning, etc.

Self-learning: learning alone.

School-learning: namely traditional class-learning style, that is, school environment, class lecturing, the teacher arranging learning resources and making, supervising and implementing learning plans and the students doing as the teacher's requirements.

Suppose one selects just one learning goal and wants to master the knowledge and skills contained in it. Denote the moment of the learner beginning to learn as time 0 , below the symbols of some factors which will be used are given.

$t$ : time.

$T$ : a moment in learning process.

$H^{*}$ : total amount of knowledge and skills contained in the goal. 
$T^{*}$ : moment that the learner completes the learning of the goal.

$H(t)$ : amount of the knowledge and skills learned by the learner at $t$.

$v(t)$ : learning velocity at $t$, the amount of knowledge and skills learned per unit time by the learner at $t$ when he learns.

$\rho(t)$ : day-learning-time-rate at $t$, which is defined by $\rho(t)=\frac{\text { Learningtime of the day at } t}{\text { Wholetime of a day }}$. belonging to (unit: hour), the whole time of a day is 24 hours. $\rho(t)$ reflects the size of time spent for one for learning in a day. By the definition, $\rho(t)$ satisfies $0 \leq \rho(t)<1$.

$p(t)$ : learning ability of a learner at $t$, the amount of knowledge and skills will be learned per unit time by the learner when he learns at $t$ under the best learning resource and strategy. It is decided by the learner's intelligence, retention, learning interest as well as external pressures, etc.

$\sigma(t)$ : learning-strategy-efficiency of a learner at $t$, the ratio of the amount of knowledge and skills learned per unit time by the learner when he learns at $t$ using his learning strategy with the amount of knowledge and skills learned using the best learning strategy under the same other conditions (suppose the ratio changes only with the change of learning strategy). $\sigma(t)$ reflects the quality of the learning methods of the learner. By the definition, $\sigma(t)$ satisfies $0 \leq \sigma(t) \leq 1$.

$s(t)$ : learning-resource-efficiency of a learner at $t$, the ratio of the amount of knowledge and skills learned per unit time by the learner when he learns at $t$ using his learning resource with the amount of knowledge and skills learned using the best learning resource under the same other conditions (suppose the ratio changes only with the change of learning resource). $s(t)$ reflects the quality of the learning resource of the learner. By the definition, $s(t)$ satisfies $0 \leq s(t) \leq 1$.

\section{MODELS OF KNOWLEDGE AND SKILl ACCUMULATION}

In learning process, the learning velocity is variable. Suppose the learner learns continuously on time interval $[0, T]$. By the knowledge of calculus [6], as the distance formula of an object moving along a straight line with varying speed over a given time interval, the amount of knowledge and skills learned by the learner on $[0, T]$ should be the integral of the learning velocity on it, namely

$$
H(T)=\int_{0}^{T} v(t) d t
$$

However, no one can learn continuously in a day without eating and sleeping. Everyone always has the time of un-learning in a day. So the process of one learning is always in the light of some separated time intervals. Thus, the amount of knowledge and skills learned by the learner on a not too short interval $[0, T]$ should be the sum of some integrals as (1). But such a formula isn't convenient to investigate learning process. To get an uninterrupted integral formula, we need a new quantity, day-learning-time-rate. By it, (1) can be rewritten as follows

$$
H(T)=\int_{0}^{T} \rho(t) v(t) d t
$$

The reasons of (2)'s holding are as follows: the change of $v(t)$ is continuous, so it has little change in a short interval, and it can be seen a constant in a day; $\rho(t)$ is day-learning-time-rate, so it is a constant in a day; hence for a given day in learning process $\rho(t)$ multiplying $v(t)$ and integrating mean that the amount of knowledge and skills learned by the learner in the learning time of the day is distributed into the whole day, i.e., the learner seems to learn all the day but the velocity drops so that the amount of knowledge and skills learned by the man in the day is unchanged (here we deem $v(t)$ as the learning velocity at $t$ if one learns no matter he really learns or not).

Equation (2)'s holding may be approximate, but for the convenience of discussion and applications it is expressed as an equation.

Let $T=T^{*}$, then $H(T)=H^{*}$ and there is

$$
H^{*}=\int_{0}^{T^{*}} \rho(t) v(t) d t
$$

If $\rho(t), v(t)$ are constants (hereafter denoted as $\rho$ and $v$ respectively), by (2) and (3) there are

$$
\begin{aligned}
H(T) & =\rho v T \\
H^{*} & =\rho v T^{*}
\end{aligned}
$$

Equation (2) is useful, but it is too simple, so it needs to improve. Apparently $v(t)$ plays a crucial role in the process of learning. So, what factors affect it? Learning velocity should be involved with learning ability, learning strategy and learning resource. Without learning resource any learning cannot be done, while learning ability does the same. And a learning will soon fall into a standstill state with a very poor learning strategy, so $v(t)$ should be expressed as follows:

$$
v(t)=p(t) \sigma(t) s(t)
$$

Substituting (6) into (2), a complete form of the accumulation process of knowledge and skill is obtained

$$
H(T)=\int_{0}^{T} \rho(t) p(t) \sigma(t) s(t) d t
$$

Substituting (6) into (3), there is

$$
H^{*}=\int_{0}^{T^{*}} \rho(t) p(t) \sigma(t) s(t) d t
$$

If $\rho(t), p(t), \sigma(t)$ and $s(t)$ are constants (represented by $\rho$, $p, \sigma$ and $s$ respectively), by (8) there is

$$
H^{*}=\rho p \sigma s T^{*}
$$

The above formulae are the mathematical models of knowledge and skill accumulating in learning process. 


\section{MODELS ANALYSIS AND DISCUSSION}

We have built some models reflecting knowledge and skill accumulation in learning process. First, we should show the correctness of these models. Following we do this work.

1) Simply speaking, (2) or (4) indicates that factors influencing the accumulation of knowledge and skill are the size of time spent for one for learning in a day and learning velocity. And the amount of knowledge and skills obtained by a learner at $T$ is decided by the two and $T$, the length of time of the learning process. This is correct obviously. Further speaking, (7) indicates that factors influencing the accumulation of knowledge and skill are day-learning-time-rate, learning ability, learning strategy and learning resource. These factors generally cover all the objective and subjective factors which influence the accumulation of knowledge and skill.

2) Equation (7) indicates that the amount of knowledge and skills learned by a learner at $T$ is the integral of his $\rho(t)$ multiplying $p(t), \sigma(t)$ and $s(t)$ over interval $[0, T]$. For a chosen goal, the amount of knowledge and skills needed to master for the learner, i.e., $H^{*}$, is fixed at the beginning. Thus by (8), to master the amount of knowledge and skills contained in the goal early or shorten the value of $T^{*}$, the learner could enhance the value of his $\rho(t), p(t), \sigma(t)$ or $s(t)$ and that enhancing their values all together can accomplish the goal of shortening $T^{*}$ more quickly.

3) Also by (7), if each of the four functions $\rho(t), p(t), \sigma(t)$ and $s(t)$ is equal to zero or close to it, the accumulation of knowledge and skill will fall into a standstill state. Thus for a learner, the best strategy of improving his velocity of knowledge and skill accumulation is improving the relatively smallest value of the four functions.

4) Equation (4) shows that the amount of knowledge and skills learned by a learner at $T$ is direct proportional to $\rho, v$ and $T$ if $\rho(t), v(t)$ are constants. Hence if day-learning-time-rate, learning velocity or the length of time of the learning process doubles, the amount of knowledge and skills learned by the learner will be doubled. If they all double, the amount of knowledge and skills learned will be octuple.

5) For a chosen goal, the amount of knowledge and skills contained in the goal is fixed. Equation (5) shows that if learning velocity is constant, to shorten the accomplishing time of the goal to half, the time used for the goal in a day must be doubled; if the time used for the goal in a day is constant, to shorten the accomplishing time of the goal to half, the learning velocity must be doubled.

6) Now let's consider those cases that the four functions take extreme values respectively, which may help us understand the models. $\rho(t)$ being equal to zero or closing to it means that the learner is too busy to learn, or has little desire or interest to learn, etc. As the time of one's life is limited, by (3) and (5) the too small value of $\rho(t)$ leads to too great value of $T^{*}$ and the goal cannot be finished (suppose $H^{*}$ is not too small). $p$ $(t)$ being equal to zero or closing to it means that the learner's ability is too poor or the goal's knowledge and skills are too difficult to him. By (6), (8) and (9) the too small value of $p(t)$ leads to too small value of $v(t)$ and too great value of $T^{*}$, and the goal cannot be accomplished. $\sigma(t)$ being equal to zero or closing to it means that the learner does not know how to learn, how to use his resources and how to make appropriate learning plans, etc. By (6) too small $\sigma(t)$ leads to too small $v(t)$, although the learner spends a lot of time for learning, the goal cannot be completed. $s(t)$ being equal to zero or closing to it means that the learner does not have basic learning resources, or that he needs some special equipments or directions in certain stage but he has not. For the lack of these resources, his $v(t)$ is quite small and the goal cannot be completed. When $\rho$ $(t), p(t), \sigma(t)$ and $s(t)$ all take the greatest values, these mean that the learner is quite clever, very diligent, good at learning and possesses very good family and society conditions. This situation is often found in self-learning. $\rho(t), p(t), \sigma(t)$ and $s(t)$ all being the greatest values lead to a result that the learner may get the amount of knowledges and skills in several years while others cannot get them in their whole life.

By 1)-6) we see that models (1)-(9) can explain learning phenomenon well, so they should be correct.

\section{Discuss SCHOOL-LEARNING AND SELF-LEARNING}

In learning styles, school-learning and self-learning are two essential learning styles. Let's discuss the advantages and disadvantages of the two.

First, we discuss school-learning. By (7) in school-learning, the learning resources usually have been configured well by the need of learning, so $s(t)$ should be 1 . In school, the teacher not only imparts knowledge and skills to his students, but also tells them how to learn. And for an experienced teacher, his pedagogy is the accumulation of long time of society and himself, thus it usually is nearly perfect. So for one of his students, if he does as his teacher's instructions, his learning method usually is the best, hence $\sigma(t)$ may take 1 . School is the specific place where students learn. Under school setting and administration system, students usually spend all the time that can be used for learning, so the value of $\rho(t)$ can be seen as the maximum. In school-learning, the teaching pattern is mainly based on class-teaching. There are many students in a class and there must be some differences in learning ability between these students, so under this situation the teacher usually arranges his teaching plan or progress by the medium ability students. Since the learning way of students in school-learning is that students follow their teachers' plans, so for a high ability student he will not get the greatest amount of knowledge and skills in a given time interval which he may get; whereas for a poor ability student he will feel difficult in learning, that is, $p(t)$ may not be the value which it should be for a pupil. Obviously this is a defect which is not easy to overcome for school-learning. Though there is this defect, the accumulation of knowledge and skill is the integral of the multiplying of $\rho(t), p(t), \sigma(t)$ and $s(t)$, and in school-learning three of them have been their maximum, while the remainder usually is not too small. This shows that school-learning is a good learning patter. Moreover, for an ordinary student the value of $p(t)$ will be its maximum. Since ordinary men take the most part of people, so we could have a conclusion that school-learning is the most efficient learning style for most people. For the basic knowledges and skills needed to master 
for people and the essential quality education, the stage of school-learning is indispensable.

Following we discuss self-learning. In self-learning, there are greater differences in learning resource between different learners, and the resource of one possessing usually is poorer than that in school, so $s(t)$ is smaller than 1 . There also are greater differences in learning strategy between different learners. Some learners' strategy may be the most efficient one whereas many learners' strategies have little efficiency. Usually the strategy of one's self-learning is not better than that in school, so $\sigma(t)$ is smaller than 1. The environment of one's self-learning usually is poorer than that in school, and self-learning also has not the efficient supervision system as that in school, so $\rho(t)$ usually is smaller than that in school. In self-learning, a learner can arrange his learning progress according to his ability and other conditions, so $p(t)$ may take the maximum. By the above analyses we see that the multiplying of $\rho(t), p(t), \sigma(t)$ and $s(t)$ in self-learning usually is much smaller than that in school for ordinary men. This shows that self-learning usually is a lower efficient learning style for people. Because there are differences in each of the functions $\rho(t), p(t), \sigma(t)$ and $s(t)$ in self-learning for different people, their multiplying has much greater differences, which means that for a fixed goal some people may complete it in one year whereas some other people cannot complete it for several years, but they complete it by the same time under school-learning.

\section{INSTRUCTIVE MEANING OF MODELS}

The built models not only can explain learning phenomenon, but also can give instructions for educators and learners. Following we use several circumstances to show it.

Consider school-learning, Section $\mathrm{V}$ said that in school-learning teachers usually arrange their teaching progress by medium ability students. Why? Let's show the reasons, the problem in it and how to deal with it. If the teacher arranges his progress by high ability students, most students in the class cannot follow the progress, so it is unfeasible. If the teacher does by poor ability students, it will influence the velocity of learning knowledge and skill of most students, so it is unsuitable. When he does by medium ability students, there are no problems for learning for the students having medium ability or greater than it, but it has a problem for poor ability students, i.e., they cannot follow this progress. To solve this problem the teacher can give some special considerations for them, such as coaching them specially, to let them follow the progress. As these students are not too many, so this progress is feasible, and the method of solving the problem is given.

Though school-learning is a better learning style, it has its defects. It does not suit the learning needs emerging from living and works in any place and at any time. It also cannot meet the learning needs of the latest knowledges and skills, and this just is the advantage of self-learning. Although self-learning usually is a poorer effective style, it is not restricted by places, settings and time, so self-learning cannot be replaced by other learning styles. Following we consider some instructive values from the models for self-learners.
For most self-learners, the aim of learning usually is to grasp some knowledge and skills so as to service himself for his life. Thus for a learner, selecting learning goal becomes very important. If the chosen goal is improper, the knowledge and skills in the goal cannot be grasped by him or cannot be grasped for a long time, thus this learning is difficult to give him satisfactory payment. By the models when choosing a goal a learner should focus on two aspects. One is that whether or not he has sufficient learning resource for the goal. If the learning of the goal needs some special equipments or platforms but he has not, this goal should not be selected. The other is whether he is interested in this goal and whether he has talent on it. If he likes the goal and his talent is consistent with the goal, the goal can be selected.

In the learning process, if one finds his learning stops, i.e., a lot of time runs off, but the new learned knowledge or skill is little, it is the time for him to look for the reason. By the models the reason may be that the knowledge is too difficult to him, his learning method is improper, or he needs some special equipments or instructions but he has not. Knowing the reason and dealing with it, the learning will go on.

Besides for a self-learner, it usually is a good idea that in the learning process, especially in the primary stage of it, he could spend some time to improve his learning methods and learning resource so as to accomplish the learning of the goal more quickly.

\section{CONCLUSIONS}

This paper builds several mathematical models on knowledge and skill accumulating in learning progress to study learning phenomenon from quantitative perspective. These models not only can explain learning phenomenon, but also can analyze the advantages and disadvantage of different learning styles, and can give some enlightenments for learning and education. Thus it opens up a new path of studying learning and education.

\section{REFERENCES}

[1] X.Z. Li, and Y. Geng, "From survival to sustainable development: the research of the evolution of lifelong learning concept: based on UNESCO's report," Tsinghua Journal of Education, 2017, vol. 38, pp.94-100. (In Chinese)

[2] M. Zhu, and Z.M. Gao, "Global development retrospect and future prospect of lifelong education,lifelong learning and learning society," Open Education Research, 2014, vol. 20, pp.50-66. (In Chinese)

[3] J. Wang, and W.D. Chen, "Review the essence of blended learning from embodied cognition perspective," Journal of distance education, 2016, pp.68-74. (In Chinese)

[4] Y.G. Zhou, Q.S. Sang, "Summary of the Home and Overseas Research on Independent Learning," Journal o f Anhui Institute of Education, 2007, vol.25, pp.100-104. (In Chinese)

[5] R. D. Johnson, S. Hornik, and E. Salas, "An empirical examination of factors contributing to the creation of successful e-learning environments." International Journal of Human-Computer Studies, 2008,vol. 66, pp.356-369.

[6] R. T. Smith, and R. B. Minton, Calculus, 2rd ed., China: Higher Education Press, 2004. 